\title{
Comparing conditioning regimens for autologous stem cell transplantation in lymphoma patients
}

Guldane Cengiz Seval', Deniz Goren Sahin², Ender Akcaglayan Soydan³, Tulay Ozcelik2, Ayla Gokmen³, Zafer Gokgoz², Pervin Topcuoglu', Onder Arslan 1. Muhit Ozcan', Mutlu Arat4

Ankara University School of Medicine, Department of Hematology, Ankara, TURKEY; 2 Bilim University School of Medicine, Department of Hematology, stanbul, TURKEY; ${ }^{3}$ Private Medicana Hospital, Hematology Clinic, Ankara, TURKEY; ${ }^{4}$ Florence Nightingale Hospitals, Hematology Clinic, Istanbul, TURKEY.

\section{Background}

Autologous hematopoietic stem cell transplantation (HSCT) is a potentially curative therapeutic approach for relapsed/refractory lymphomas. Although carmustine, etoposide, cytarabine, and melphalan (B-EAM) is the most commonly used conditioning regimen for high-dose therapy (HDT) before auto-HSCT, to date, there is no published prospective randomized study comparing different types of conditioning regimen. Here, we aim to compare between B-EAM and alternative alkylator -EAM backbone based regimen on the potential toxicities and survival in lymphoma patients.

\section{Materials \& Methods}

Patients with relapsed refractory lymphoma, who underwent autologous HSCT between 2012-2017, were evaluated from three different highly active transplant institutions in Turkey. One hundred eighty adult ymphoma patients (age: 18-65 years) with a follow up time at least 6 months were included. Patients with B-EAM $(n=139)$ high dose chemotherapy were compared with patients having received non-BEAM regimen; T- thiotepa $(T)$-EAM $(n=16)$, Bendamustine $(B e)-E A M$ $(n=12)$, Lomustine $(\mathrm{L})$-EAM $(\mathrm{n}=13)$ using the following matching factors: age, gender, lymphoma subtype, disease status at transplant, toxicity, overall survival, and causes of death

Table 1.Demographic and clinical characteristics of lymphoma patients who had received either BEAM or Non-BEAM conditioning.

\begin{tabular}{|c|c|c|c|}
\hline Patients & B-EAM & Non-BEAM & P value \\
\hline Median follow-up (months) & 29.2 & 6.5 & $<0.0001$ \\
\hline $\begin{array}{l}\text { Age at HSCT, median (SD) } \\
\text { (years) }\end{array}$ & $46.6( \pm 15.1)$ & $47.7( \pm 14.3)$ & 0.67 \\
\hline Gender male n (\%) & $87(62.6 \%)$ & $22(53.7 \%)$ & 0.36 \\
\hline $\begin{array}{l}\text { Entities n (\%) } \\
\text { NHL } \\
\text { HL }\end{array}$ & $\begin{array}{l}94(67.6 \%) \\
45(32.4 \%)\end{array}$ & $\begin{array}{l}30(73.2 \%) \\
11(26.8 \%)\end{array}$ & 0.56 \\
\hline $\begin{array}{ll}\text { Disease status at transplant, } \mathrm{n} \\
\text { (\%) } \\
\text { CR1 } \\
\text { CR2 } \\
\text { PR } \\
\text { Refractory }\end{array}$ & $\begin{array}{l}20(15.9 \%) \\
24(19.0 \%) \\
53(42.1 \%) \\
29(23.0 \%)\end{array}$ & $\begin{array}{l}6(14.6 \%) \\
6(14.6 \%) \\
20(48.8 \%) \\
9(22.0 \%)\end{array}$ & 0.74 \\
\hline Mucositis, n (\%) & $69(49.6 \%)$ & $31(75.6 \%)$ & 0.01 \\
\hline Renal, n (\%) & $6(4.3 \%)$ & $2(4.8 \%)$ & 0.92 \\
\hline $\begin{array}{l}\begin{array}{l}\text { Gastrointestinal system, } \mathbf{n} \\
(\%)\end{array} \\
\text { \%) }\end{array}$ & $71(51.0 \%)$ & $32(78.0 \%)$ & 0.002 \\
\hline 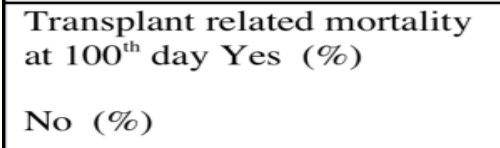 & $\begin{array}{c}6(5.5 \%) \\
104(94.5 \%)\end{array}$ & $\begin{array}{c}4(11.4 \%) \\
31(88.6 \%)\end{array}$ & 0.25 \\
\hline $\begin{array}{l}\text { Secondary malignancies } \\
\text { Yes }(\%) \\
\text { No }(\%)\end{array}$ & $\begin{array}{c}5(3.6 \%) \\
134(96.4 \%)\end{array}$ & $\begin{array}{c}0(0 \%) \\
39(100 \%)\end{array}$ & 0.58 \\
\hline $\begin{array}{l}\text { Last disease status } \\
\text { CR (\%) } \\
\text { Refractory\&Relapse (\%) }\end{array}$ & $\begin{array}{l}41(74.5 \%) \\
14(25.5 \%)\end{array}$ & $\begin{array}{c}22(73.3 \%) \\
8(26.7 \%)\end{array}$ & 0.9 \\
\hline
\end{tabular}

Figure 1. Kaplan-Meier survival curves of BEAM vs alternative alkylator-EAM (Non-BEAM) group (log-rank analysis, $p=0.2$ )
Kaplan-Meier Survival Analysis

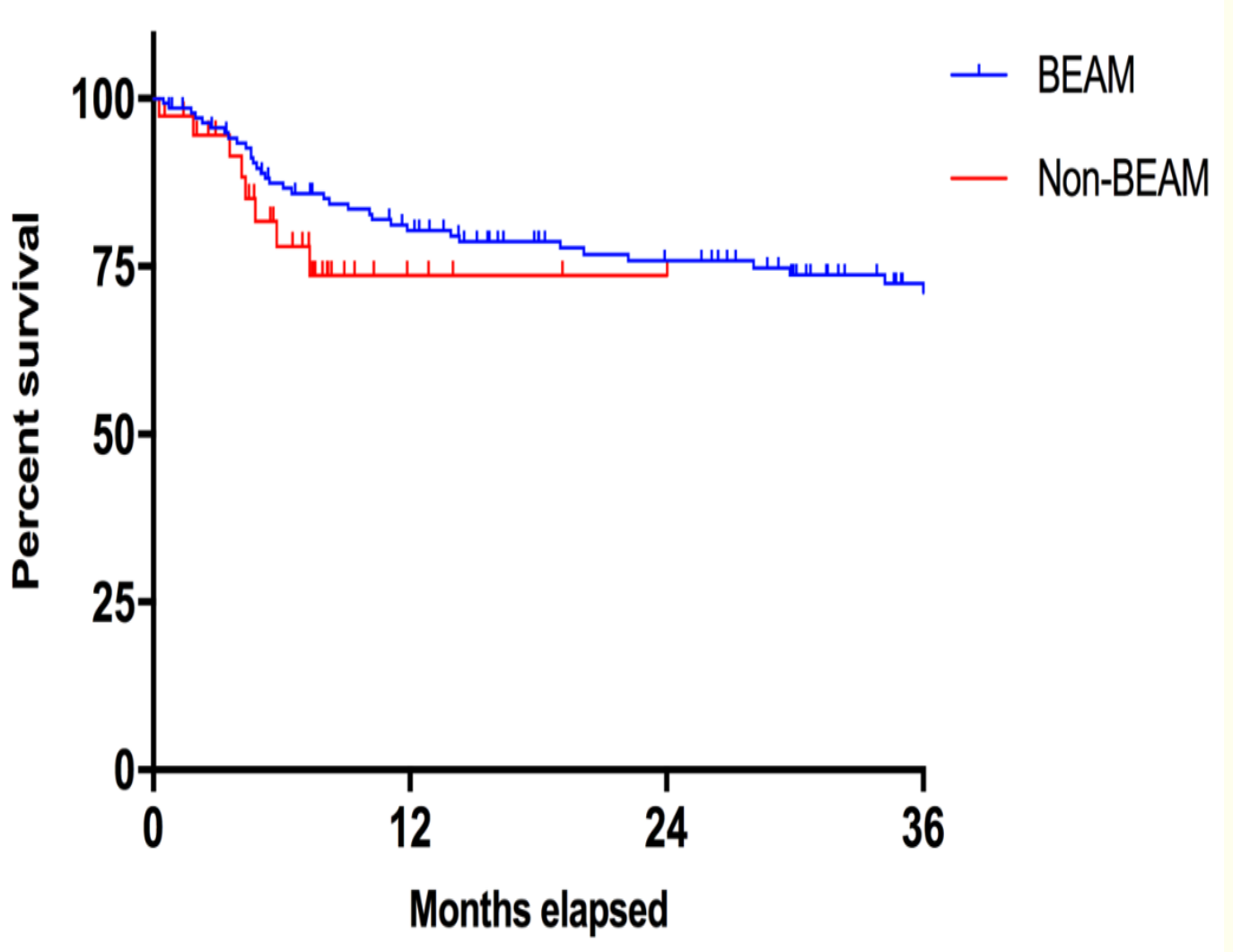

\section{Results}

Totally 180 patients (109 males, 71 females) were identified: 139 patients and 41 patients were treated with BEAM and non-BEAM regimen, respectively. Patients' baseline characteristics are detailed in Table 1. There was no statistically significant difference between mean age and gender between groups. With regards to toxicities among groups; BEAM group had a significantly lower rate of mucositis and gastrointestinal toxicity than non-BEAM group ( $p=0.01$ and $p=0.002$, respectively). Renal toxicity did not show difference among groups $(p=0.92)$. Although BEAM group showed a lower rate of transplant related mortality at $100^{\text {th }}$ day ( $5.5 \%$ vs. $11.4 \%)$, the difference did not reach to statistical significance. Estimated mean survival time for BEAM and Non-BEAM was 52.1 \pm 2.5 months and $18.8 \pm 1.5$ months, respectively $(p=0.2$, log-rank test) (Figure 1). The significant difference in median follow/up times has to be taken into account.

\section{Discussion}

Our study showed that EAM backbone (nonBEAM) conditioning regimens with alternative alkylating agents have similar results compared to B-EAM regimen in terms of efficacy, and toxicity. Prospective randomized controlled studies with large number of patients are warranted to find out the optimal backbone combination, which may establish as a standard regimen for HSCT in lymphoma patients. 